\title{
Intersatellite Links and Relative Navigation: Pre-conditions for Formation Flights with Pico- and Nanosatellites
}

\author{
Michael Marszalek* Oliver Kurz** Maximilian Drentschew* \\ Marco Schmidt*** Klaus Schilling*** \\ * ZfT - Zentrum für Telematik e.V., Gerbrunn, Germany ( e-mail: \\ m.marszalek-m.drentschew@telematik-zentrum.de). \\ ** Fraunhofer Institute for Integrated Circuits IIS ,Nürnberg, Germany \\ (e-mail: oliver.kurz@iis.fraunhofer.de) \\ *** Chair of Computer Science VII - Robotics and Telematics, \\ University Würzburg, Germany (e-mail: \\ schmidt.marco-schi@informatik.uni-wuerzburg.de)
}

\begin{abstract}
:
A group of satellites can be classified as a constellation, formation or cluster. Multiple coordinated satellites are described as a formation, if a closed-loop control is adjusted, thus providing a coordinated motion control on basis of their relative positions to preserve the topology (Schilling [2009]). This technology enables various application scenarios, for example, interferometry or in-orbit servicing and it is subject to multiple technological requirements related to formation flying, limited resources or scientific payloads. In the framework of the feasibility study NaKoFo, this paper discusses the potential benefits, drawbacks and problems associated with intersatellite links and relative navigation with pico- and nanosatellites. The requirements of an intersatellite link are analyzed based on frequency, modulation, antenna, power and network protocol. A short overview of adequate communication systems for pico- and nanosatellites is also presented. Technologies for relative position determination are presented and discussed regarding their applicability in small satellite systems as well as their requirements on an intersatellite link.
\end{abstract}

\section{INTRODUCTION}

In the past years, various autonomous formation flying missions have been successfully demonstrated. TanDEM$\mathrm{X}$ was launched in the first half of 2010 as a technology mission, designed to fly in a closely controlled formation with TerraSAR-X. This mission facilitates a new area in space borne radar remote sensing. Both almost identical spacecrafts will generate a consistent global digital elevation model of the earth with an unprecedented accuracy. Each satellite has a mass around $1200 \mathrm{~kg}$ which implies high launch and development costs.

In contrast, the application of pico- and nanosatellites has significant scientific and cost advantages over using large, heavy satellites. It enables research institutions to reduce costs while implementing technology demonstration missions in space. This trend will take advantage of the advances in Micro-Electro-Mechanical Systems (MEMS) in the upcoming years. An example for a formation flying mission could be the observation of volcanic ash clouds (see Figure 1), done by a closed controlled formation of picosatellites. It can be expanded to include a greater extent of satellites, as envisioned by NASA's ANTS's Mission (Curtis et al. [2000]).

The implementation of autonomy implies a minimal dependence on ground stations and so intersatellite links must be used to allow satellites to share their individual

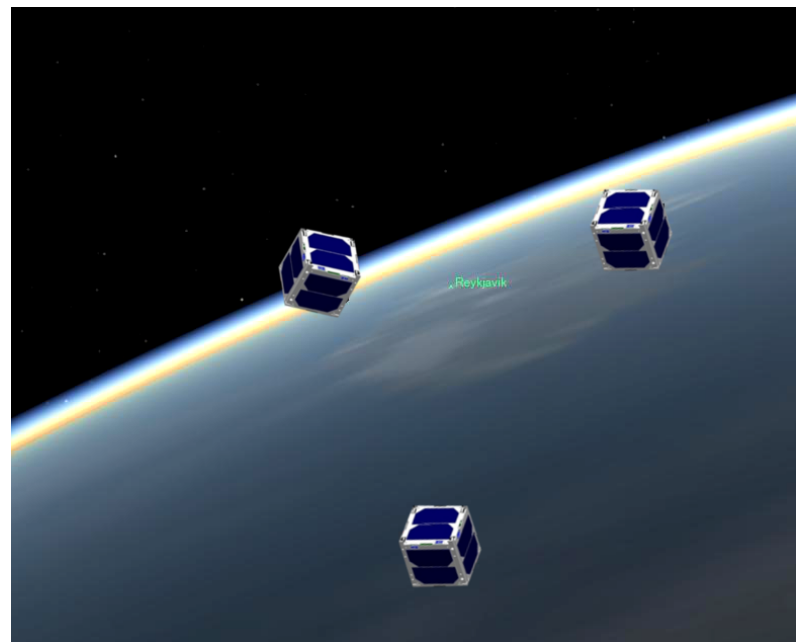

Fig. 1. Observation of a volcanic ash cloud with picosatellites

information and use their combined resources to achieve a more complex goal (Kusza and Paluszek [2000]). The shared information include relative poses, attitudes or status information. An Inter-Satellite Link (ISL) defines a two-way communication path between satellites and provides flexibility in the space segment implementation. A position determination system independent of frequent groundstation contact is necessary not only for establish- 


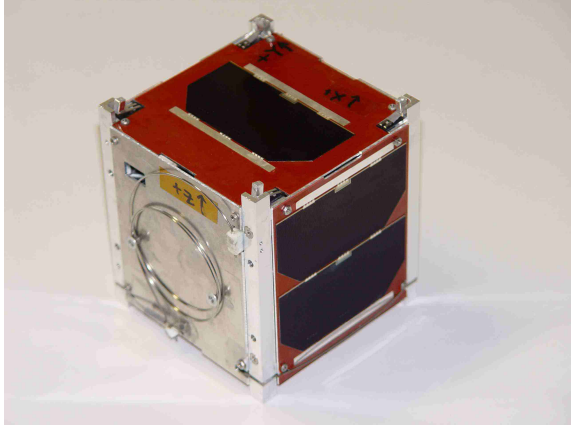

\section{Fig. 2. UWE-1}

ing a communication link but also for almost any application which ought to be fulfilled by the satellite formation (Bauer et al. [1999]).

The realization of the communication system acts in accordance with a mission scenario due to specific requirements in terms of frequency, modulation, communication distance, data rate, antenna technology or network protocol. For example, TanDEM- X and TerraSAR-X are flying in a closely controlled formation with distances between 250 and $500 \mathrm{~m}$. In contrast, scheduled formation missions like FAST will deal with relative distances between 1 and $1225 \mathrm{~km}$ (Delft [2010]). Formation flying also demands different control accuracies. An overview of orbit control requirements for past and future mission with its dependence on the operation distance is presented in (Gill et al. [2001]). Small satellites without orbit control suffer from drift caused by various perturbations such as Earth's geophysical forces or solar radiation pressure. Thereby the topology of a satellite group and thus the network connectivity and relative distances change over time. A mobile ad-hoc network (MANET) with its reconfiguration capabilities promises significant increases in robustness in case of network topology changes (Drentschew et al. [2011] and Bridges and Vladimirova [2008]). An effective space network will also require the optimization of terrestrial wireless protocols to space to satisfy the needs of autonomous distributed satellites. (Magness and Plancke [2006]) proposed different network classes (e.g. landerrover, microsensor-lander network) and the use of terrestrial IEEE 802.11 wireless protocols. A full, in-depth presentation of all requirements is beyond the scope of this paper. Rather, we will focus on the intersatellite link and relative navigation system. In section 2 , we will briefly describe some pico- and nanosatellite missions, followed by an overview of useful communication and relative navigation systems in section 3 and 4 .

\section{STATE-OF-THE-ART}

The following overview introduces examples for pico- and nanosatellite missions. So far, formation flying has been demonstrated by large satellites like TanDEM-X but the main objective of this paper is the identification of usable communication and relative navigation systems for picoand nanosatellites. There were many successful picosatellite missions in the past years. A survey with detailed information is listed in (Klofas et al. [2008]).

UWE-1 was the first German picosatellite, built by the University of Würzburg (Schmidt and Zeiger [2006]). The communication subsystem is based on a modified PR430 transceiver, normally used by radio amateurs for data transmission via packet radio. It supports a baud rate of 1200/9600 and applies the AX.25 communication protocol. The main scientific objective was the optimization of the Internet Protocol (IP) parameters in adaption to the measured space environment. Furthermore cross layer optimizations have been analyzed between AX.25 and higher protocols (i.e. IP, HTTP) (Schilling [2009]). Despite limited resources of picosatellites, it was possible to complete telecommunication experiments and demonstrate the availability of IP on a CubeSat. The implementation of UWE-1 and its successor UWE-2 provided important know-how for upcoming missions towards formation flying (Schmidt et al. [2008]).

CanX-4\&5 are nanosatellites of the Spaceflight Laboratory (SFL) at the University of Toronto. This mission will establish the core technologies necessary for formation flying endeavors at any scale, while capitalizing on the lowcost nature and rapid design cycle of nanosatellites (Callibot et al. [2005], Orr et al. [2007] and Orr et al. [2008]). The precursors CanX-1 and CanX-2 are already operating in orbit. CanX-2 proved a S-Band communication system in combination with a patch antenna in order to enable an intersatellite link for CanX-4\&5, which is capable of transmitting/receiving $10 \mathrm{kbps}$ to a maximum range of $5 \mathrm{~km}$. The relative navigation system is based on carrierphase differential GPS techniques and a navigation control algorithm with accuracies less than $10 \mathrm{~cm}$ for seperation distances between 50 and $1000 \mathrm{~m}$. The determination of the absolute position is stated with an accuracy around $1 \mathrm{~m}$.

GeneSat-1, MAST and KySat-1 are similar picosatellite missions with regard to the communication systems. All satellites are using a S-Band Commercial Off-The-Shelf (COTS) transceiver of Microhard Systems Inc. for data transmission (Microhard Systems Inc. [2010]). GeneSat1and KySat-1 are equipped with an amateur band and Sband communication system. The UHF/VHF subsystem of KySat-1 enables the satellite to downlink data and receive commands from amateur band ground stations. The additional S-band transceiver is the MHX2400 spread spectrum radio, manufactured by Microhard Systems Inc. While GeneSat-1 was launched on 11 December 2006, the KySat-1 mission is currently scheduled on November 2010.

\section{SURVEY OF COMMUNICATION SUBSYSTEMS}

Transceivers are an important part of the communication system. The amount and reliability of transmitted data depends on the implemented transceiver. The design of an entire transceiver out of individual components is subject to various challenges. Building a custom communication subsystem allows tighter control of requirements and specifications, and encourages the next generation of students to learn about building small RF circuits (Klofas et al. [2008]). These transceivers are usually less successful, which is why the adaption of a commercial off-the-shelf transceiver is preferred in the scope of upcoming missions. Many COTS transceivers would have significant difficulties functioning in space. The reduction of mass and volume, limited power resources or active thermal dissipation are 
Table 1. Overview of adequate transceivers

\begin{tabular}{|c|c|c|c|c|c|}
\hline Transceiver & Frequency & Data Rate & Distance & Output Power & Mass \\
\hline MHX-2420 & $2,4-2,5 \mathrm{GHz}$ & $\leq 230.4 \mathrm{kbps}$ & $50 \mathrm{~km}$ & $20-30 \mathrm{dBm}$ & $55 \mathrm{~g}$ \\
\hline MHX-920A & $902-928 \mathrm{MHz}$ & $\leq 230.4 \mathrm{kbps}$ & $100 \mathrm{~km}$ & $20-30 \mathrm{dBm}$ & $55 \mathrm{~g}$ \\
\hline MHX-425 & $400-450 \mathrm{MHz}$ & $\leq 230.4 \mathrm{kbps}$ & $100 \mathrm{~km}$ & $20-30 \mathrm{dBm}$ & $80 \mathrm{~g}$ \\
\hline Stensat & $144-146 / 435-438 \mathrm{MHz}$ & $1200 \mathrm{bps}$ & $100 \mathrm{~km}$ & $6-10 \mathrm{dBm}$ & $\mathrm{N} / \mathrm{A}$ \\
\hline
\end{tabular}

some examples for these difficulties. The transceivers presented in the following, have flown on several missions and demonstrated the usability for space applications. Table 1 lists adequate transceivers with its physical parameters. The combination with an antenna enables higher communication distances. Microhard Systems Inc. develops various transceivers which differ in frequency, communication distance or mass for example.

MHX-2420 operates in the industrial, scientific and medical (ISM) radio band at speeds up to $230.4 \mathrm{kbps}$. It is significantly faster than the older MHX-2400. An improved sensitivity for greater link margins can be enabled by using the "slow mode" limited to $19.2 \mathrm{kbps}$. The transceiver's predecessor, MHX2400 has flown with GeneSat-1, MAST and KySat-1. For example, the MAST inspector satellites are equipped with a Microhard radio in combination with a monopol antenna. (Klofas et al. [2008]) stated that more than $2 \mathrm{MB}$ of telemetry have been downloaded from one inspector satellite. Another successful demonstration of a S-Band downlink was done by GeneSat-1. The satellite, equipped with a patchantenne, provided around $500 \mathrm{kB}$ of payload data.

The Frequency Hopping Wireless Modems MHX-920A and MHX-425, designed for the UHF band, feature robust, high speed, low latency and secure data communications. Both transceivers support an adjustable data rate, distances up to $100 \mathrm{~km}$, depending on data rate and line-ofsight, and a low power consumption in Sleep Mode. MHX425 was the primary on-board communication system of the picosatellite ITUpSat-1, launched 2009. An advantage of the MHX-920A is a lower mass which is an important factor for pico- and nanosatellites.

The Stensat radio is based on the Motorola MC13136 receiver and MC13175 transmitter. The uplink (ground to satellite) operates in the VHF band at a carrier frequency between 144 and $146 \mathrm{MHz}$. The AFSK modulation scheme allows a data rate of 1200 baud (i.e. 1200 symbols/second). The downlink operates in the UHF band at a frequency between 435 and $438 \mathrm{MHz}$. The operation mode can be switched between 1200 AFSK and 9600 baud FSK. Both, uplink and downlink use the AX.25 protocol for packet data transmission. It periodically transmits telemetry and was part of the communication system of the first Colombian picosatellite Libertad-1, launched 2007. The primary ground station at the university did not work during the launch campaign, and due to a rotor failure just after launch, no uplink attempts were made (Klofas et al. [2008]). The telemetry includes internal side panel and microcontroller temperatures and was transmitted every 10 minutes (Mike Rupprecht [2010]).

The listed transceivers have been analyzed in terms of distance, mass, frequency or data rate. Other transceivers flown on CubeSats in space include the Alinco DJ-C4 and DJ-C5 (Klofas et al. [2008]). The Stensat Group and
Microhard System Inc. develop transceivers for CubeSats which have been proved in space. The adaption of these commercial off-the-shelf modems simplifies the design of the communication system. So far, none of the presented modems have been used for intersatellite links but the successful missions demonstrated its serviceability.

\section{RELATIVE NAVIGATION}

Traditionally external observations with the help of ground based measurements were used for orbit determination of satellites in space, e.g. using Radio Detection and Ranging $(R A D A R)$ for flight path determination of satellites and all flying objects in the near surroundings of earth. This information is made available by North American Aerospace Defense Command (NORAD) in the form of Two Line Elements (TLE) datasets including all important orbit parameters necessary for tracking. The achievable accuracy is dependent on the refresh period and therefore on a present communication link if this data were to be present on a satellite. For a highly autonomous and adaptive relative position determination system as required for the application of formation flying, the sole usage of this data source is not appropriate and insufficient.

Laser measurements for long ranges as well as various optical methods for short distances, e.g. Photonic Mixer Devices (PMD) cameras, can be used for distance determinations with high performance and accuracy on conventional space vehicles.

\subsection{Laser technologies}

Laser technologies for distance measurements are very accurate even for high ranges and could already be successfully deployed on some satellites. Systems based on this technology are already available in small sizes and are also used on mobile robots e.g. in form of laser scanners for obstacle detection, collision avoidance or simultaneous localization and mapping. In contrast to simple distance measurements, laser scanners can also be used for the construction of a 3D image of the environment but their obligatory pan-/tilt and positioning mechanics can be quite complex and therefore demanding in terms of requirements on the satellite platform.

Indeed, the usage of laser scanners which only deliver distance information and therefore no complete 3D relative position are hardly appropriate for small satellites. One important reason is the very high requirement on an attitude control of the satellite. Also, the position of the other satellite must be known to a good degree in the forefront of the distance measurements, in order to be able to acquire a pointing of the target satellite. Additional hardware accompanying the distance measurement system must be equipped on the satellite along with a nonnegligible, necessary laser optics which defeats the purpose 
of a highly integrated system, which would be desired on small satellites with their minor availability of space and power. An example for a laser scanner is Hokuyo UTM30LX (Sensor [2010]), which is adequate for the use on mobile robots for navigation tasks. What impedes the application on small satellites is the rather high energy consumption of up to $12 \mathrm{~W}$.

\subsection{Binocular cameras}

Optical monocular cameras, which deliver visual information or optical flow patterns, have been common on spacecrafts for a long time. Their use is not solely restricted to inspection and observation tasks. Camera systems are used for docking maneuvers, especially for non-autonomous or partly autonomous operations. The operation range for these cases is limited to a few meters. The disadvantage of not directly accessible depth information when generating $2 \mathrm{D}$ picture material can be alleviated by the use of size relation estimations using the knowledge about already known objects in the camera picture, e.g. an already recorded geometry is measured and thereby a distance can be derived indirectly (Uber and Doherty [1988]).

Binocular cameras, where two partial optics separated by a fixed distance observe the same object from different angles, deliver - like the human eyes - a depth information, which can also be gained if the geometry of the observed object is unknown. Therefore, a 3D picture of the environment can be created (Capurro et al. [1993]). If these camera systems are present, they can be used for relative localization, albeit with limited range and accuracy. (Rovira Más et al. [2009]) states $20 \mathrm{~m}$ as an achievable, useful range for a lens distance of $20 \mathrm{~cm}$ where usable stereo pictures for robotic control can be derived. Current developments for distance determination show promising approaches of algorithmic data handling (Tao et al. [2010], Hao and Kang [2009]). However, current implementations are always very restricted in their applicability for very small satellites. One problem is e.g. the sensitive and elaborate optics and mechanics which can only be partially miniaturized, especially because of the physically necessary distance between the lenses as the operational range or the accuracy of the measurement is proportional to the inverse distance between the lenses (Rovira Más et al. [2009]). Possibly also the target object, in this case another satellite, must be actively illuminated which negatively increases the energy consumption of the whole system. In conclusion, binocular cameras show a promising field of application, particularly if optical systems on the satellite are obligatory for other reasons, anyway. However, an implementation of such a system at the current time is largely hindered by the too demanding restrictions of small satellites.

\subsection{D-time-of-flight-Cameras}

These very compact camera systems deliver direct distance measurements for each pixel using a time-of-flight procedure. The reflections are measured by a sensor which can pixel wise determine the distance to an object by measuring the phase difference between sent and received signals. Especially interesting for the application in space flights is the fact that the relative distance and attitude of

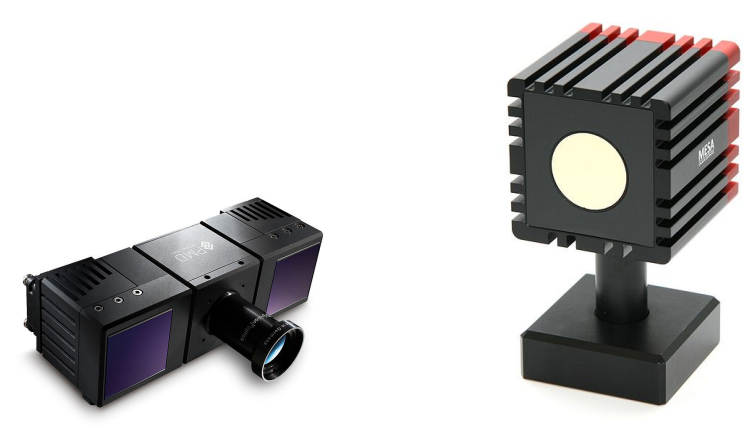

Fig. 3. 3D-Time-of-flight (TOF) cameras: left) PMD Technologies Wikipedia [2010a], right) MESA Imaging AG Wikipedia [2010b]

a target satellite can be directly gained from a 3D depth picture. (Ringbeck et al. [2007]) gives an overview of this technology.

This technology is independent of external reference points (Imaging AG [2010]). Moreover, it is energy-saving as there are neither scan cycles nor is additional computing power necessary for the computation of a 3D scenery. The geometry of big objects, e.g. a satellite that needs to be inspected, can be measured without a necessary base length increase like e.g. on binocular cameras. A further advantage for applicability on satellites is the innecessity of movable parts. The 3D time-of-flight camera is a comparably young technology for which first COTS products are available since 2005 (PMDTechnologies GmbH [2010]) which shows that there should be further potential for innovation.

There are restrictions which necessitate further technological developments: The resolution of current systems (e.g. 200x200 pixel) is limited but there are no technical reasons why this aspect cannot be improved in the long run. It is even possible to combine measurements from high-resolution cameras with the measurement data (Sauer et al. [2010]). This is especially interesting when the quality of data must be improved, e.g. for creating a detailed picture during inspection tasks. A further field of research covers the potential range of measurements. With increasing distance an adequately strong illumination of the target must be guaranteed with the help of modulated, optical signals. A commercially available 3Dtime-of-flight camera with a resolution of $176 \times 144$ pixel and an operational range of $10 \mathrm{~m}$ consumes about $12 \mathrm{~W}$ (Imaging AG [2010]). While the size and the mass of this model does nearly fulfill the requirements of picosatellites, the current power consumption is still critical in terms of a practical implementation of this device for the use of relative navigation purposes.

\subsection{Global Navigation Satellite System (GNSS)}

The generic working principle for GNSS, e.g. Global Positioning System (GPS), is the measurement of the duration of signal propagation.

The easiest way of gaining a relative positioning with GNSS is the calculation of the difference of two independently derived absolute positions. This does not pose any high demands on the receiver and the achievable accuracy is in the range of $15-20 \mathrm{~m}$ as also for absolute position 
Table 2. Comparison table of merits and shortcomings of different relative navigation approaches. Marked on a subjective, relative scale from "++" meaning very good, over "o" meaning indifferent to "- _" meaning very bad compared to the best in each category. Flexibility regarding

possible application scenarios.

\begin{tabular}{|c|c|c|c|c|c|c|}
\hline & cost & size \& weight \& power & range & reliability & accuracy & flexibility \\
\hline NORAD-RADAR + TLE & ++ & ++ & ++ & - & -- & -- \\
\hline laser technologies & -- & -- & + & 0 & ++ & - \\
\hline binocular cameras & + & $\mathrm{o}$ & -- & - & + & + \\
\hline 3D time-of-flight cameras & $\mathrm{o}$ & - & - & + & + & ++ \\
\hline GNSS & ++ & + & ++ & + & + & + \\
\hline Radio based ranging & ++ & + & + & + & $\mathrm{o}$ & ++ \\
\hline
\end{tabular}

determination using a single receiver. Another approach is the differencing of the range measurements as they are conducted in the receivers (pseudoranges). This corrects common errors like inaccurate or faulty orbits or clocks of the GNSS satellites. The relative accuracy achievable in real-time is around $10 \mathrm{~m}$ (Highsmith and Axelrad [1999]).

A big improvement in accuracy is achieved by including carrier phase measurements. Using double differencing of carrier phases, the achievable real-time accuracy is around $5 \mathrm{~cm}$ (Ebinuma [2002]). The highest exactness can be achieved by using so called Real-Time-Kinematic (RTK) methods. This principle resolves the ambiguity of carrier phase determination and a subsequent position measurement by using exclusive phase measurements is possible. RTK enables accuracies of $1 \mathrm{~cm}$ (Busse [2003], Leung and Montenbruck [2005]), at least in post-processing.

For the mentioned accuracies in the range of a few $\mathrm{cm}$, not only improved algorithms are necessary. Also, range and phase measurements must be very accurate and reliable. Outliers in the range estimation, as well as cycle slips in the phase measurement have to be eliminated or reliably detected. Depending on the seperation distance of the small satellites, and therefore the distance between the GNSS receivers, precise GNSS ephemeris information are necessary. The demands on the accuracies of the ephemeris data increases with the base length as an error in the absolute position decreases the relative position estimation.

As an example, the ISL bandwidth requirement for a relative navigation subsystem, based on a GNSS module on two satellites, is given as $1 \mathrm{kByte} / \mathrm{s}$ up to $48 \mathrm{kByte} / \mathrm{s}$ for a high-update rate Multi-GNSS receiver.

The use of optical technologies, as presented, is theoretically also possible on small satellites but they do not work in an omnidirectional way and therefore require high demands on the attitude and orbit control of the satellites. Furthermore, parameters like electrical power consumption, mass and thermal issues are so far not tailored for the application on small satellites. In contrast, a GNSS receiver can gather an absolute position information without external information - except for the presence and visibility of corresponding GNSS satellites. The same receiver can also be used for relative positioning by using the difference range measurements. Thus, it is possible to use this technology for both purposes which is especially helpful on small satellites with their limited resources. The integration of a GNSS device for relative navigation on small satellites provides an integrated system for absolute and relative positioning which - in comparison - shows good performance. Using multiple GNSS antennas, even an absolute attitude of the satellite can be gained without any further sensors.

\subsection{Radio based ranging}

Radio based positioning systems for formation flying take advantage from the fact: Pico- and Nanosatellites have limited resources regarding size and power. A combined communication and navigation system enables an intersatellite link as well as a relative navigation system for formation flying. Ranging labels distance measurements which are a prerequisite for localisation. The accuracy and achievable distance of the relative ranging system depends on the measuring technique (e.g. ToF, ToA, RSSI) as well as the communication protocol (e.g. WLAN, UWB).

A simplified qualitative comparison of all mentioned technologies is shown in table 2 .

\section{CONCLUSION}

This paper considers two main requirements for the implementation of formation flying with small satellites. We presented an overview of various transceivers for the implementation of an intersatellite link in terms of mass, distance, frequency or data rate. Relative navigation is a second important requirement for formation flying. Various technologies have been introduced and discussed regarding their applicability.

The ISL needs to guarantee a minimum data rate for the transmission of information between satellites in a formation, e.g. for a differencing GNSS system, allowing higher positioning accuracies than single-point measurements.

The proposed relative navigation technologies have different accuracies, operational ranges and requirements. For example, a laser range finder demands an AOCS. In contrast, GNSS or radio based ranging show potential for a broad operation range and proofed to be the technologies of choice for formation flying missions with picoand nanosatellites. Formation flying demands further key enabling technologies such as formation control algorithms or a propulsion system which need to be identified in future works. It will open the doors to numerous future missions and applications in space.

\section{ACKNOWLEDGEMENTS}

The authors would like to acknowledge the support of the Bavarian Ministry for Economic Affairs, Infrastructure, Transport and Technology and all project partners for this research. 


\section{REFERENCES}

F. H. Bauer, K. Hartman, J. P. How, J. Bristow, D. Weidow, and F. Busse. Enabling spacecraft formation flying through spaceborne gps and enhanced automation technologies. In Proc. of ION GPS-00 Conference, Nashville, TN, USA, September 1999.

C. P. Bridges and T. Vladimirova. Dual core system-on-achip design to support inter-satellite communications. In Proc. of the NASA/ESA Conference on Adaptive Hardware and Systems, 2008.

F.D. Busse. Precise formation-state estimation in low Earth orbit using carrier differential GPS. PhD thesis, Stanford University, 2003.

E. P. Callibot, C. C. Grant, D. D. Kekez, and R. E. Zee. Formation Flying Demonstration Missions Enabled by Canx Nanosatellite Technology. In Proc. of the 19th AIAA/USU Conference on Small Satellites, SSC05-VII6, Salt Lake City, Utah, USA, August 2005.

C. Capurro, F. Panerai, E. Grosso, and G. Sandini. A binocular active vision system using space variant sensors: Exploiting autonomous behaviors for space application. In International Conference on Digital Signal Processing, Nicosia, Cyprus. Citeseer, 1993.

S. Curtis, J. Mica, J. Nuth, G. Marr, M. Rilee, and M. Bhat. Ants (autonomous nano technology swarm): An artificial intelligence approach to asteroid belt resource exploration. In Proc. of 51st International Astronautical Congress, Rio de Janeiro, Brazil, October 2000.

TU Delft. Fast overview, 2010. URL http:// www.Ir.tudelft.nl/live/pagina.jsp?id= da8b4974-e3ef-480d-ab04-76c7ae46bba0\&lang=en.

M. Drentschew, M. Marszalek, M. Schmidt, F. Zeiger, M. Sauer, and K. Schilling. Results of a requirements study for mobile ad-hoc networks of small satellites. In 62nd International Astronautical Congress, Cape Town, South Africa, October 2011. to appear.

T. Ebinuma. Precision spacecraft rendezvous using Global Positioning System- An integrated hardware approach. Dissertation Abstracts International, 2002.

E. Gill, M. Steckling, and P. Butz. Gemini: A milestone towards autonomous formation flying. In ESA Workshop on On-Board Autonomy, ESTEC, Noordwijk, October 2001.

M. Hao and C. Kang. Object location technique for binocular stereo vision based on scale invariant feature transform feature points. Journal of Harbin Engineering University, page 06, 2009.

D. Highsmith and P. Axelrad. Relative state estimation using GPS flight data from co-orbiting spacecraft. In ION GPS'99, pages 401-409, 1999.

MESA Imaging AG. SwissRanger SR4000 Data Sheet. datasheet, 08 2010. URL http://www.mesa-imaging. $\mathrm{ch} / \mathrm{dlm}$.php?fname=pdf/SR4000\_Data\_Sheet.pdf.

B. Klofas, J. Anderson, and K. Leveque. A survey of cubesat communication systems. In Technical Report. California Polytechnic State University, 2008.

K. L. Kusza and M. A. Paluszek. Intersatellite links: Lower layer protocols for autonomous constellations. In Princeton Satellite Systems, November 2000.

S. Leung and O. Montenbruck. Real-time navigation of formation-flying spacecraft using global-positioningsystem measurements. Journal of Guidance Control and Dynamics, 28(2):226-235, 2005.
R. Magness and P. Plancke. Esa tec-e wireless technology dossier, annex a: An assessment of wireless proximity networks for space applications. In Proc. of the 9th ESA Workshop on Advanced Space Technologies for Robotic and Automation (ASTRA), ESTEC, Noordwijk, Netherlands, November 2006.

Microhard Systems Inc. Microhard systems, 2010. URL http: //www . microhardcorp. com.

Mike Rupprecht. Cubesat libertad-1, 2010. URL http:// www.dk3wn.info/sat/afu/sat/_libertad.shtml.

N. G. Orr, J. K. Eyer, B. P. Larouche, and R. E. Zee. Precision Formation Flight: The CanX-4 and CanX-5 Dual Nanosatellite Mission. In Proc. 21st Annual AIAA/USU Conference on Small Satellites, Logan, Utah, August 2007.

N. G. Orr, J. K. Eyer, B. P. Larouche, and R. E. Zee. Precision Formation Flight: The CanX-4 and CanX-5 Dual Nanosatellite Mission. In Proceedings 2008 Small Satellites Systems and Services - The $4 S$ Symposium, Rhodos, Greece, May 2008.

PMDTechnologies GmbH. PMDTechnologies, 082010. URL http: //www.pmdtec. com/.

T. Ringbeck, T. Möller, and B. Hagebeuker. Multidimensional measurement by using 3-D PMD sensors. Advances in Radio Science, 5:135-146, 2007.

F. Rovira Más, Q. Wang, and Q. Zhang. Design parameters for adjusting the visual field of binocular stereo cameras. Biosystems Engineering, 2009.

M. Sauer, F. Leutert, and K. Schilling. Occlusion Handling in Augmented Reality User Interfaces for Robotic Systems. Proceedings-ISR/ROBOTIK 2010, 2010.

K. Schilling. Networked Distributed Pico-satellite Systems for Earth Observation and Telecommunication Applications. In IFAC Workshop Aerospace Guidance, Navigation and Flight Control Systems - AGNFCS, Samara, June-July 2009

M. Schmidt and F. Zeiger. Design and implementation of in-orbit experiments for the pico satellite uwe-1. In $I A F$ StudentConference, Valencia, IAC-06-E2.1.07, 2006.

M. Schmidt, K. Ravandoor, O. Kurz, S. Busch, and K. Schilling. Attitude determination for the pico-satellite uwe-2. In Proceedings IFAC World Congress, Seoul, pages 14036-14041, 2008.

SOKUIKI Sensor. "Scanning range finder" UTM-30LX, 2010. URL http://www.hokuyo-aut.jp/02sensor/ 07scanner/utm_30lx.html.

LIU Tao, YU Zhong qing, and MA Qian li. Target Distance Computing Based on Parallel Binocular Stereo Vision. Journal of Qingdao University (Natural Science Edition), 1, 2010.

G.T. Uber and MF Doherty. Real-time object determination for space robotics. In 1988 IEEE International Conference on Robotics and Automation, 1988. Proceedings., pages 1320-1321, 1988.

Wikipedia, 2010a. URL http://de.wikipedia.org/ w/index .php?title=Datei:PMDvision_CamCube. jpg\&filetimestamp $=20090525084158$.

Wikipedia, 2010b. URL http://de.wikipedia. org/w/index.php?title=Datei :TOF_Kamera . jpg\&f iletimestamp $=20081029140035$. 